\title{
Parides klagesi (Ehrmann) redescoberto no Brasil (Lepidoptera, Papilionidae, Troidini) ${ }^{1}$
}

\author{
Olaf H. H. Mielke ${ }^{2,3} \&$ Mirna M. Casagrande ${ }^{2,3}$
}

${ }^{1}$ Contribuição n ${ }^{0} 1664$ do Departamento de Zoologia, Universidade Federal do Paraná

${ }^{2}$ Departamento de Zoologia, Universidade Federal do Paraná, Caixa postal 19020, 81531-980 Curitiba-PR, Brasil. omhesp@ufpr.br, mibras@ufpr.br ${ }^{3}$ Pesquisador $\mathrm{CNPq}$

\begin{abstract}
Parides klagesi (Ehrmann) rediscovered in Brazil (Lepidoptera, Papilionidae, Troidini). Parides klagesi (Ehrmann, 1904), a species described from Venezuela, has its geographical distribution confirmed to Brazil (Pará and Maranhão).
\end{abstract}

KEYWORDS. Lepidoptera; neotropical; geographical distribution; Brazil.

RESUMO. Parides klagesi (Ehrmann) redescoberto no Brasil (Lepidoptera, Papilionidae, Troidini). Parides klagesi (Ehrmann, 1904), uma espécie descrita da Venezuela, tem a sua ocorrência confirmada para o Brasil (Pará e Maranhão).

PALAVRAS CHAVE. Lepidoptera; neotropical; distribuição geográfica; Brasil.

A diversidade da fauna brasileira está longe de ser conhecida. Surpresas como novas espécies e principalmente registros de novas ocorrências de espécies conhecidas, justificam esta afirmativa. Do ponto de vista da lepidopterofauna, enormes são os espaços do Brasil onde não se tem registros, mas também há outros muito bem conhecidos e de onde advêm surpresas, como no caso que segue.

Ao visitar o Sr. Pierre Jauffret em Santo Antônio de Tauá, Pará, cidade próxima de Belém, uma das áreas mais bem conhecidas do Brasil, verificou-se a existência de uma série de Parides klagesi (Ehrmann, 1904) em sua coleção. Esta é a menor espécie do gênero e sempre foi um enigma para os lepidopterologistas, principalmente para os especialistas em Papilionidae.

Este artigo tem como finalidade ilustrar e oferecer novos dados de distribuição da espécie, confirmando-a para o Brasil.

\section{MATERIAL}

A presente comunicação baseia-se em material das coleções Pierre Jauffret (Santo Antônio de Tauá, Pará, Brasil), "The Natural History Museum" (Londres, Inglaterra) e Departamento de Zoologia, Universidade Federal do Paraná, conforme mencionado adiante, nos resultados.

\section{HISTÓRICO}

A descrição original baseia-se em uma fêmea, conseqüentemente o holótipo, coletada por Edw. A. Klages em Suapure, Venezuela, cidade às margens do Rio Caura, afluente da margem direita do Rio Orinoco. O holótipo está na coleção Ehrmann, no Carnegie Museum of Natural History, Pittsburgh, Pennsylvania, USA.Após a descrição, como Papilio klagesi (Ehrmann, 1904: 215) e de acordo com D‘Almeida (1966: 49), a espécie havia sido mencionada nove vezes, porém há mais uma citação de Talbot (1932), omitida no seu catálogo. Rothschild \& Jordan (1906: 453) mencionam mais três fêmeas coletadas em fevereiro e março de 1899 em Suapure, a localidade típica, figurando uma delas. Jordan (1907: 14) repete os mesmos dados. Boullet \& Le Cerf (1912: 4) mencionam que não há exemplares no "Museum National d"Histoire Naturelle" de Paris. Ehrmann (1918: 82) menciona um macho da mesma localidade (um erro de identificação - vide Holland, 1927). Kaye (1920: 352), em uma tabela de distribuição geográfica dos Papilionidae americanos, menciona a localidade típica. Holland (1927: 318) tece comentários sobre o holótipo fêmea e diz que o macho mencionado por Ehrmann (1918: 82) é um exemplar pequeno de Parides neophilus ecbolius (Rothschild \& Jordan, 1906) (citada no gênero Papilio Linnaeus, 1758). Bryk (1930: $325,624)$, em catálogo, menciona partes das referências anteriores. Talbot (1932: 189) é o primeiro autor a descrever o verdadeiro macho proveniente de Orinoco, Venezuela, recebido da firma R. Krueger, Leipzig, Alemanha; este exemplar está depositado no "The Natural History Museum", Londres. F.M. Brown (1950: 41) cita como ocorrendo no noroeste da América do Sul. Munroe (1961: 45, 49) a inclui no gênero Parides Hübner, [1819]. D“Almeida (1966: 49) a menciona como Battus (Parides) klagesi. D‘Abrera (1981: 14, 15) ilustra 1 macho e 1 fêmea (um erro de identificação - vide Tyler et al., 1994). Tyler et al. (1994: 44, 50, 70, 177, 178, 185, 211, 323,360, pl. 56, figs O e 30) ao revisarem toda a família Papilionidae das Américas, mencionam que ocorre em floresta estacional, que as formas imaturas são desconhecidas e que a espécie ocorre no Pará e Maranhão e em outras áreas, além da localidade típica, na Venezuela, sem no entanto oferecer maiores detalhes; afirmam que os adultos voam rapidamente próximo ao chão, na floresta ou beiras floridas e ilustram parte da genitália masculina (harpe), além de figurar o macho e a fêmea; mencionam também que os exemplares figurados por D'Abrera (1981) são anões de Parides anchises thelios (Gray, [1853]). 


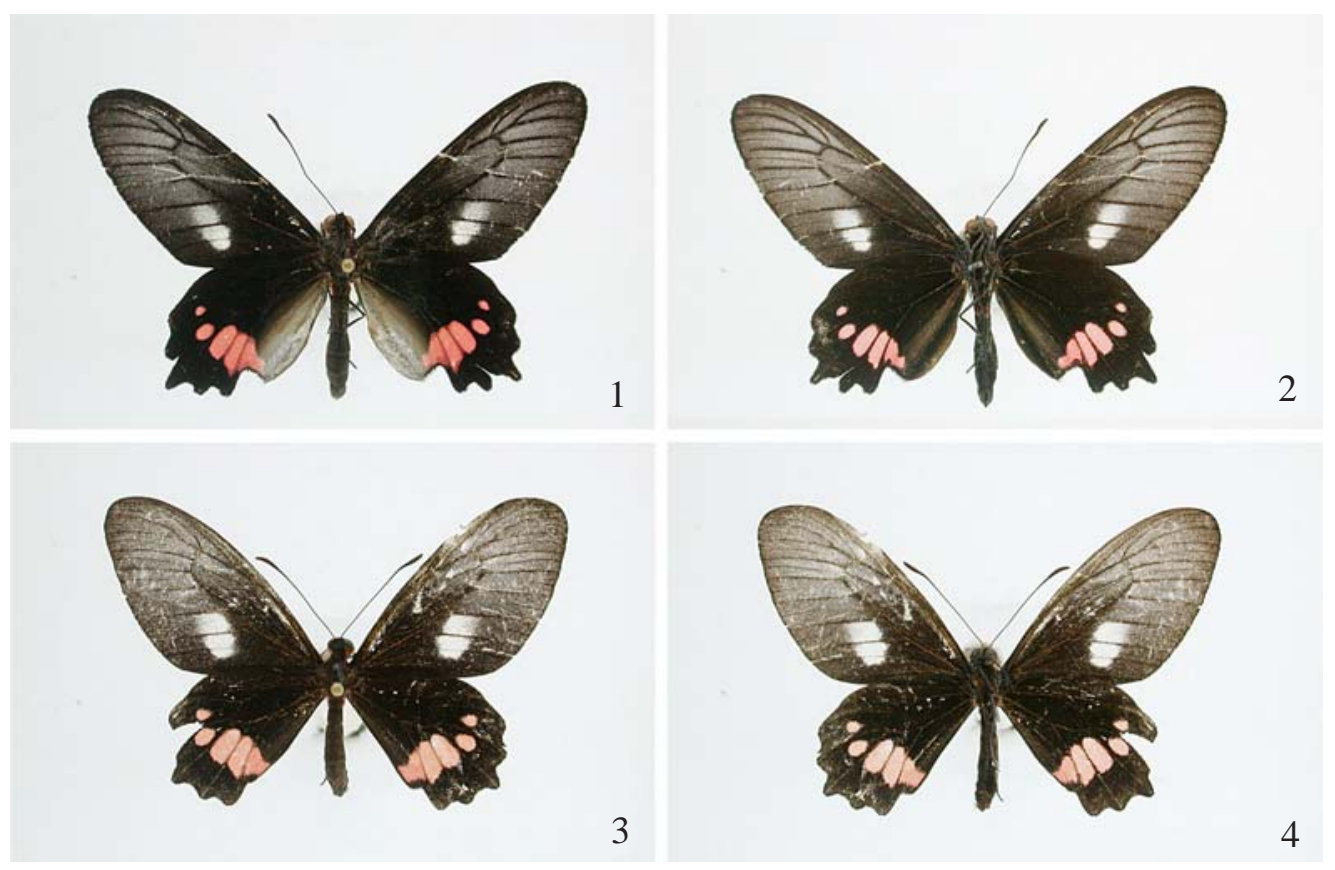

Figs. 1-4. Parides klagesi (Ehrmann). 1-2:Macho, faces dorsal e ventral, 10-IX-2002, Reserva Sonho Azul, Santo Antônio de Tauá, Pará, Brasil, P. Jauffret leg. 3-4: Fêmea, faces dorsal e ventral, 29-X-2002, Reserva Sonho Azul, Santo Antonio do Tauá, Pará, Brasil, P. Jauffret leg.

Assim o sendo, confirma-se pela primeira vez a sua ocorrência no Brasil, mais precisamente na cidade de Santo Antônio de Tauá, no Estado do Pará, onde os exemplares citados neste artigo foram coletados pelo Sr. Pierre Jauffret na área de sua propriedade, atualmente a RPPN "Reserva Sonho Azul".

Os exemplares coincidem com as figuras de Tyler $e t a l$. (1994), no entanto, os machos do Pará possuem a mancha branca da face dorsal da asa anterior pouco mais larga, como pode ser observado na comparação entre as figuras apresentadas por Tyler et al. (1994) e as figuras 1-4 deste artigo. Ao todo foram coletados seis machos e quatro fêmeas nos meses de setembro, outubro e novembro, sugerindo ser uma espécie univoltina, pois o Sr. Jauffret mora na reserva onde ocorre a espécie e observa regularmente os lepidópteros. Segundo o Sr. Jauffret, a espécie voa entre 6:30 e 9:00 horas de modo lento, típico das demais espécies de Parides Hübner, [1819], a uma altitude entre três e cinco metros do solo em áreas úmidas dentro da mata primária. Machos marcam território ao longo de uma abertura na mata de até $40 \mathrm{~m}$, aproximadamente. Como esta espécie foi redescoberta no Pará, a procedência Montes Áureos, Maranhão, de onde provém um exemplar depositado na coleção do "The Natural History Museum", Londres, deve ser correta. Esta região se localiza no noroeste do Maranhão com as seguintes coordenadas, de acordo com K. Brown (1979): $02^{\circ} 25^{\prime}$ latitude sul e $46^{\circ} 02^{\prime}$ longitude oeste.

As datas de captura dos exemplares são como seguem: 7IX-2000 (2 fêmeas), 10-IX-2000 (1 macho), 15-IX-2000 (1 macho, 1 fêmea), 16-IX-2000 (1 macho), 20-IX-2000 (1 macho), 1-XI2000 (1 macho), 10-IX-2002 (1 macho) e 29-X-2002 (1 fêmea), sendo que os últimos dois estão depositados na coleção do Departamento de Zoologia da Universidade Federal do Paraná com os números DZ 10.184 e DZ 10.338, respectivamente, e os exemplares machos de 16-IX-2000 e de 15-IX-2000 foram doados pelo Sr. Pierre Jauffret ao Museu Paraense Emilio Goeldi, Belém, Pará. Os demais exemplares conhecidos são: o [holó]tipo fêmea, Suapure, Venezuela (Carnegie Museum of Natural History), 1 macho, "Orinoco", Venezuela, ex coleção Joicey, "neallotype" por Talbot (1932); 3 fêmeas de II-1899, 13-II-1899, 11-III-1899, Suapure, Venezuela, Klages leg.; 1 fêmea, 22-IV-1987, Rio Caura, Javillal, Venezuela, H. Skinner leg.; 1 fêmea Montes Áureos, Maranhão, Brasil, T. Belt leg.; todos no "The Natural History Museum”, Londres.

Agradecimentos. Os nossos profundos reconhecimentos ao Sr. Pierre Jauffret, amigo e pesquisador autônomo de borboletas, pela doação de um casal desta espécie para o Departamento de Zoologia, UFPR, assim como ao Dr. Gerardo Lamas, Museo de Historia Natural, Lima, Peru, pelas informações dos exemplares da coleção do "The Natural History Museum", Londres, Inglaterra.

\section{REFERÊNCIAS}

Boullet, E. \& F. L. Le Cerf, 1912. Catalogue de la collection de lépidoptères du Muséum national d'Histoire naturelle de Paris. 1. Famille Papilionidae. Paris, Imprimérie Nationale. IV $+47 \mathrm{pp}$

Brown, F. M. 1950. The American Papilios. Lepidopterists' News, New Haven, 4(4/5): 39-41, (6/7): 63-67.

Brown Jr., K. S. 1979. Ecologia geográfica e evolução nas florestas neotropicais. São Paulo, Universidade Estadual de Campinas. XXXI + 265 pp., Apendices, 120 pp.

Bryk, F. 1930. Papilionidae. Lepidopterorum Catalogus, Berlin, 35: 1-55; 37: 57-509; 39: 511-676.

D`Abrera, B. 1981. Butterflies of the Neotropical Region. Part 1. 
Papilionidae \& Pieridae. East Melbourne, Lansdowne Editions. XVI + 172 pp.

D‘Almeida, R. F. 1966. Catálogo dos Papilionidae americanos. São Paulo, Sociedade Brasileira de Entomologia. 366 pp.

Ehrmann, G. A. 1904. New forms of exotic Papilionidae. Entomological News, Philadelphia, 15: 214-215.

Ehrmann, G. A. 1918. New exotic Papilios. Lepidoptera, Boston, 2: 82-84.

Holland, W. J. 1927. The Lepidoptera named by George A. Ehrmann. Annals of the Carnegie Museum, Pittsburgh, 17: 299-364.

Jordan, K. 1907-1908. 1. Familie: Papilionidae, Segelfalter, pp. 11-51, pls 1-17. In: A. Seitz (ed.). Die Gross-Schmetterlinge der Erde 5. Stuttgart, Alfred Kernen Verlag. 1141 pp., 194 pls.
Kaye, W. J. 1920. A geographical table to show the distribution of the American Papilios. Novitates Zoologicae, Tring, 26: 320-355.

Munroe, E. G. 1961. The classification of the Papilionidae (Lepidoptera). Canadian Entomologist, Ottawa, 93, Supplement 17: 1-51.

Rothschild, L. W. \& H. E. K. Jordan 1906. A revision of the American Papilios. Novitates zoologicae, Tring, 13: 411-752, pls. 4-9.

Talbot, G. 1932. New species and forms of Lepidoptera from South America. Bulletin of the Hill Museum, London, 4: 189-197.

Tyler, H. A.; K. S. Brown Jr. \& K. H. Wilson 1994. Swallowtail butterflies of the Americas. A study in biological dynamics, ecological diversity, biosystematics, and conservation. Gainesville, Scientific Publishers. 376 pp., 100 pls. 